\title{
LA RESPONSABILIDAD PENAL DE LOS INDUSTRIALES EN LOS JUICIOS POSTERIORES DE NÚREMBERG Y SUS ENSEÑANZAS PARA EL CASO COLOMBIANO
}

Omar Huertas Díaz Iván Ricardo Morales Chinome Carolina Amaya Sandoval Paula Andrea Cruz Tibaduiza Sandra Johanna Arévalo Fonseca

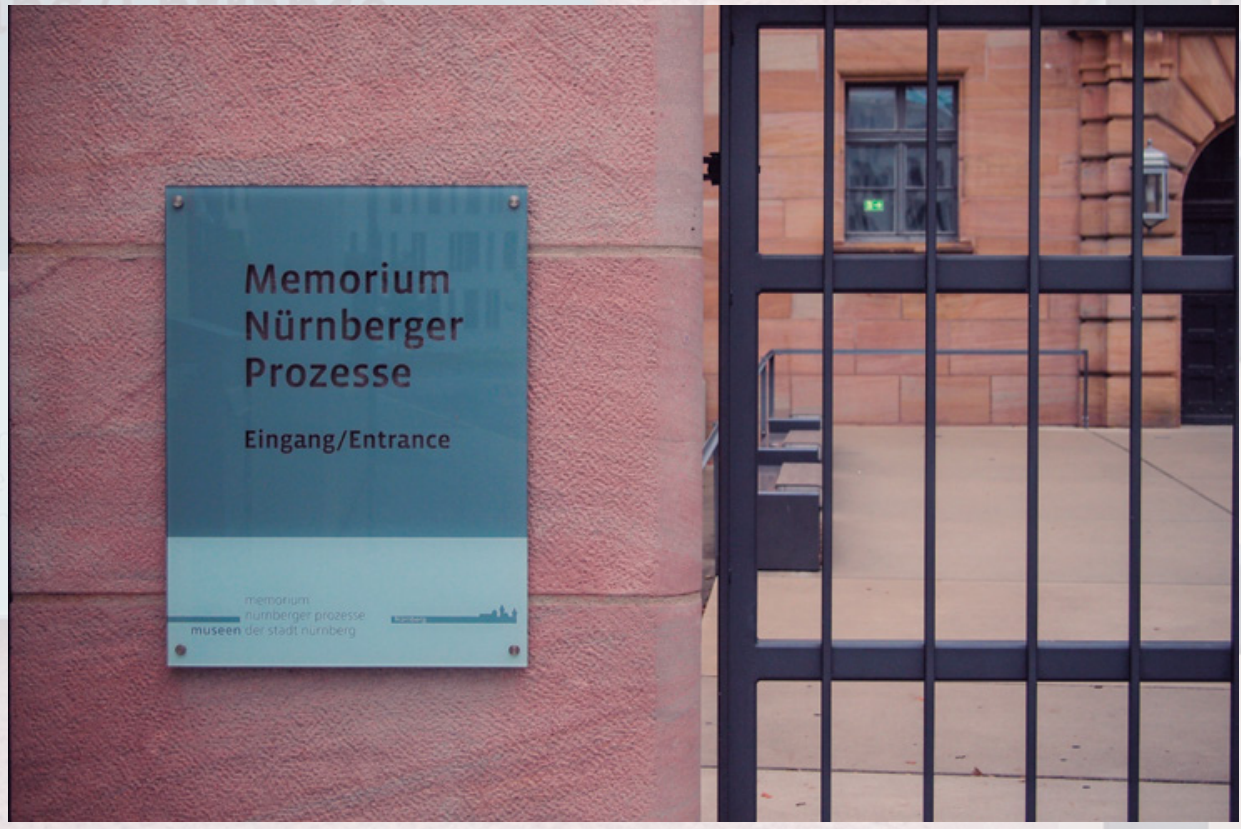





\title{
LA RESPONSABILIDAD PENAL DE LOS INDUSTRIALES EN LOS JUICIOS POSTERIORES DE NÚREMBERG Y SUS ENSEÑANZAS PARA EL CASO COLOMBIANO*
}

\author{
Omar Huertas Díaz \\ Iván Ricardo Morales Chinome \\ Carolina Amaya Sandoval \\ Paula Andrea Cruz Tibaduiza \\ Sandra Johanna Arévalo Fonseca \\ Universidad Nacional de Colombia
}

\section{Resumen}

Los doce juicios posteriores de Núremberg fueron personales, adelantados contra criminales nacionalsocialistas por el Tribunal Militar Nacional Americano. Los procesos se dividieron en profesiones y gremios dentro del Gobierno nazi. En los casos Flick, I. G. Farben y Krupp fueron condenados grandes industriales alemanes por su participación en la comisión de crímenes de guerra y de lesa humanidad durante la Segunda Guerra Mundial, pues fue revelada la relación entre la industria y el régimen. Además, se establecieron estándares para investigar y juzgar este tipo de macrocriminalidad y determinar la responsabilidad penal de directores de firmas, de acuerdo con el derecho penal internacional. Estos criterios pueden ser importantes para estudiar casos como el colombiano, los que se investigan a miles de civiles que pertenecieron a corporaciones y empresas que estuvieron involucradas en la ejecución de tales crímenes durante el conflicto armado.

Artículo de investigación desarrollado dentro del proyecto "Tratamiento de la responsabilidad de mando de los agentes del Estado colombiano y de las FARC-EP en la comisión de crímenes de guerra, contemplada en el Acuerdo final para la terminación del conflicto y la construcción de una paz estable y duradera", del grupo de investigación Red internacional de política criminal sistémica UN, financiado por la Facultad de Derecho, Ciencias Políticas y Sociales de la Universidad Nacional de Colombia. Código Hermes 46355. 
Palabras clave: juicios posteriores de Núremberg, derecho penal internacional, empresarios, industriales, crímenes de guerra, crímenes de lesa humanidad, Jurisdicción Especial para la Paz.

Los autores: Omar Huertas Díaz, doctor en Derecho y Ciencias de la Educación, investigador senior de la Facultad de Derecho, Ciencias Políticas y Sociales de la Universidad Nacional de Colombia. ORCID: 0000-0002-8012-2387. Correo electrónico: ohuertasd@unal.edu.co

Iván Ricardo Morales Chinome, magíster en Derecho con profundización en Sociología y Política Criminal, investigador del grupo de investigación "Red internacional de política criminal sistémica extrema ratio UN", de la Universidad Nacional de Colombia. ORCID: 0000-0002-9319-3993. Correo electrónico: irmoralesc@unal.edu.co

Carolina Amaya Sandoval, magíster en Derecho Penal, coordinadora de investigaciones de la "Red internacional de política criminal sistémica extrema ratio UN". ORCID: 0000-0002-9319-3993. Correo electrónico: caramayasan@unal.edu.co

Paula Andrea Cruz Tibaduiza, estudiante de Derecho, integrante de la "Red internacional de política criminal sistémica extrema ratio UN”. ORCID: 0000-0002-9319-3993. Correo electrónico: pcruzt@ unal.edu.co

Sandra Johanna Arévalo Fonseca, magíster en Criminología y Victimología, investigadora y docente de la Fundación Universitaria San Mateo. ORCID: 0000-0003-2259-4316. Correo electrónico: sjhoannaa@sanmateo.edu.co

Recibido: 8 de agosto de 2021; evaluado: 23 de septiembre de 2021; aceptado: 27 de octubre de 2021. 


\title{
THE CRIMINAL LIABILITY OF INDUSTRIALS IN THE SUBSEQUENT TRIALS OF NUREMBERG AND ITS LESSONS FOR THE COLOMBIAN CASE
}

\author{
Omar Huertas Díaz \\ Iván Ricardo Morales Chinome \\ Carolina Amaya Sandoval \\ Paula Andrea Cruz Tibaduiza \\ Sandra Johanna Arévalo Fonseca \\ Universidad Nacional de Colombia
}

\begin{abstract}
The twelve Nuremberg trials established criminal liability against national socialists by the American National Military Tribunal. The trials were split according to occupation and rank within the Nazi government. In the Flick, I. G. Farben, and Krupp cases, important German industrialists were convicted for their involvement in the commission of war crimes and crimes against humanity during the Second World War. These processes revealed the relationship between the German industry and the Nazi regime. In addition, standards were established to investigate and prosecute this type of macrocrime and determine the criminal responsibility of business owners, in accordance with international criminal law. These criteria may be important to study cases such as the Colombian one, in which thousands of civilians who belonged to corporations and companies involved in the execution of such crimes during the armed conflict are being investigated.
\end{abstract}

Keywords: subsequent Nuremberg Trials, international criminal law, businessmen, industrialists, war crimes, crimes against humanity, Special Jurisdiction for Peace. 
About the authors: Omar Huertas Díaz, PhD in Law and Educational Sciences, senior researcher at the Faculty of Law, Political and Social Sciences of the Universidad Nacional de Colombia. ORCID: 0000-0002-8012-2387.E-mail: ohuertasd@unal.edu.co

Iván Ricardo Morales Chinome, MA in Law with specialization in Sociology and Criminal Policy, researcher of the "Red Internacional De Política Criminal Sistémica Extrema Ratio UN" Research Group, Universidad Nacional de Colombia. ORCID: 0000-0002-9319-3993. E-mail: irmoralesc@ unal.edu.co

Carolina Amaya Sandoval, MA in Criminal Law, research coordinator of the "Red Internacional De Política Criminal Sistémica Extrema Ratio UN” Research Group. ORCID: 0000-0002-9319-3993. E-mail: caramayasan@unal.edu.co

Paula Andrea Cruz Tibaduiza, law student, member of the "Red Internacional De Política Criminal Sistémica Extrema Ratio UN” Research Group. ORCID: 0000-0002-9319-3993. E-mail: pcruzt@ unal.edu.co

Sandra Johanna Arévalo Fonseca, MA in Criminology and Victimology, researcher and professor at the Fundación Universitaria San Mateo. ORCID: 0000-0003-2259-4316. E-mail: sjhoannaa@ sanmateo.edu.co

Received: August 8, 2021; evaluated: September 23, 2021; accepted: October 27, 2021. 


\title{
A RESPONSABILIDADE CRIMINAL DOS INDUSTRIAIS NOS JULGAMENTOS SUBSEQUENTES DE NUREMBERG E SUAS LIÇÕES PARA O CASO COLOMBIANO
}

\author{
Omar Huertas Díaz \\ Iván Ricardo Morales Chinome \\ Carolina Amaya Sandoval \\ Paula Andrea Cruz Tibaduiza \\ Sandra Johanna Arévalo Fonseca \\ Universidad Nacional de Colombia
}

\section{Resumo}

Os doze julgamentos de Nuremberg estabeleceram responsabilidade criminal contra o nacionalsocialismo pelo Tribunal Militar Nacional Americano. Os processos foram divididos de acordo com a ocupação e a classificação dentro do governo nazista. Nos casos de Flick, I. G. Farben e Krupp foram condenados por grandes industriais alemães por seu envolvimento na prática de crimes de guerra e crimes contra a humanidade durante a Segunda Guerra Mundial, já que a relação entre a indústria e o regime foi revelada. Além disso, foram estabelecidas normas para investigar e processar esse tipo de macrocriminalidade e determinar a responsabilidade criminal dos diretores das empresas, de acordo com o direito penal internacional. Esses critérios podem ser importantes para estudar casos como o colombiano, que investigam milhares de civis que pertenciam a corporações e empresas envolvidas na execução desses crimes durante o conflito armado.

Palavras-chave: julgamentos posteriores de Nuremberg, direito penal internacional, empresários, industriais, crimes de guerra, crimes contra a humanidade, Jurisdição Especial para a Paz. 
Os autores: Omar Huertas Díaz, doutor em Ciências de Direito e Educação, pesquisador sênior da Faculdade de Direito, Ciências Políticas e Sociais da Universidad Nacional de Colombia. ORCID: 0000-0002-8012-2387.E-mail: ohuertasd@unal.edu.co

Iván Ricardo Morales Chinome, Mestre em Direito com aprofundamento em Sociologia e Política Criminal, pesquisador do grupo de pesquisa "Rede Internacional de Política Criminal Sistêmica Extrema Ratio UM", da Universidad Nacional de Colombia. ORCID: 0000-0002-9319-3993. E-mail: irmoralesc@unal.edu.co

Carolina Amaya Sandoval, Mestre em Direito Penal, coordenadora de pesquisa da "Rede Internacional de Índice de Política Criminal Sistêmica Extrema Ratio UN". ORCID: 0000-0002-9319-3993. E-mail: caramayasan@unal.edu.co

Paula Andrea Cruz Tibaduiza, estudante de Direito, membro da "Rede Internacional de Índice de Política Criminal Sistêmica Extrema Ratio UN”. ORCID: 0000-0002-9319-3993. E-mail: pcruzt@ unal.edu.co

Sandra Johanna Arévalo Fonseca, Mestre em Criminologia e Vitimologia, pesquisadora e professora da Fundación Universitaria San Mateo. ORCID: 0000-0003-2259-4316. E-mail: sjhoannaa@ sanmateo.edu.co

Recebido: 8 de agosto de 2021; avaliado: 23 de setembro de 2021; aceito: 27 de outubro de 2021. 


\section{Introducción}

Tras el final de la Segunda Guerra Mundial, los representantes del régimen nacionalsocialista fueron procesados y juzgados en el Palacio de Justicia de Núremberg. El juicio principal tuvo lugar entre el 20 de noviembre de 1945 y el 1 de octubre de 1946, en contra de los principales criminales de guerra ante el Tribunal Militar Internacional.

Hubo otros juicios en las zonas ocupadas por Francia, Gran Bretaña, Estados Unidos y la Unión Soviética; sin embargo, los doce juicios posteriores de Núremberg, adelantados en la zona con ocupación estadounidense son los más conocidos. ${ }^{1}$

Estos fueron juicios personales contra otros criminales nacionalsocialistas. Los acusados eran importantes funcionarios del régimen nazi, de la Administración, del Ejército, del Poder Judicial, de la industria y de las SS. ${ }^{2}$ Su fundamento jurídico fue la Ordenanza núm. 7 del Gobierno Militar y la Ley núm. 10 del Consejo de Control Aliado del 20 de diciembre de 1945.

Los doce procesos se dividieron en profesiones y gremios dentro del gobierno nazi: el caso médico (caso 1), de Milch (caso 2), de los juristas (caso 3), de Pohl (caso 4), de Flick (caso 5), de I. G. Farben (caso 6), de los generales del Sureste, "Juicio por asesinato de rehenes" (caso 7), de RuSHA contra los promotores de la idea de pureza racial (caso 8), de los einsatzgruppen o escuadrones móviles de la muerte (caso 9), de Krupp (caso 10), de Wilhelmstrasse, "Juicio de Ministerios" (caso 11), y del OKW o alto mando de la Wehrmacht (caso 12). ${ }^{3}$

Allí fueron condenados grandes industriales alemanes. En el caso Flick (caso 5), I. G. Farben (caso 6) y Krupp (caso 10), la mayoría de los acusados eran empresarios y directores de la industria pesada. La relación entre la industria alemana y el régimen nazi fue expuesta en estos tres procesos. Además, se estableció la responsabilidad

1 Christoph Safferling, "Der Nürnberger Prozess" en Krieg und Recht. Die Ausdifferenzierung des Rechts von der ersten Haager Friedenskonferenz bis heute, eds. Martin Löhnig, Mareike Preisner y Thomas Schlemmer (Regenstauf: Rechtskultur, 2014), 96.

2 Christoph Safferling, Internationales Strafrecht: Strafanwendungsrecht-Völkerstrafrecht-Europäisches Strafrecht (Heidelberg: Springer, 2011), 55.

3 George Andoor, "Das Nürnberger Tribunal vor 70 Jahren-Teil 2 Faires Verfahren anhand der Grundsätze eines neuen Völkerstrafrechts", Zeitschrift für das Juristische Studium 5, 8 (2015): 474, http://www.zjs-online. com/dat/artikel/2015_5_939.pdf (acceso mayo 8, 2021). 
penal de los grandes industriales: 42 del consorcio Flick, la sociedad I. G. Farben y el consorcio Krupp fueron juzgados por crímenes de guerra y de lesa humanidad.

Estos procesos fueron criticados en su momento. Incluso, algunos partidarios de los juicios de Núremberg se sintieron decepcionados con las sentencias del Tribunal Militar, ${ }^{4}$ pero a pesar de ello, su importancia para el desarrollo actual del derecho penal internacional es notable.

La investigación académica presentada es hermenéutica, en la cual se interpretó la jurisprudencia del Tribunal Militar Nacional estadounidense en Núremberg en los procesos adelantados contra los industriales (Flick, I. G. Farben y Krupp) y se analizó la posibilidad de emplear algunas de aquellas subreglas jurisprudenciales en el caso colombiano. En la primera etapa se realizó una revisión bibliográfica de las sentencias y de la literatura académica sobre el tema; en la segunda, se analizaron las decisiones y se extrajeron las subreglas determinadas por el tribunal, y en la tercera, se elaboraron la propuesta y las conclusiones.

Este artículo tiene como objetivo proporcionar una visión general de los juicios posteriores de Núremberg contra los industriales y examinar algunas tesis que pudieran ser útiles para el caso colombiano, en particular el juzgamiento de los empresarios que participaron en la comisión de crímenes de guerra y contra la humanidad en el conflicto armado interno.

Para cumplir con dicho objetivo, este documento se divide en cuatro partes. En la primera se describe cada uno de los tres casos contra industriales; en la segunda, se describen los criterios legales con los cuales el Tribunal Militar condenó a los acusados; la tercera explica las críticas más importantes contra estos juicios y en la última se presenta su importancia para el caso colombiano.

\section{Los juicios posteriores de Núremberg contra industriales}

En Flick, I. G. Farben y Krupp, los propietarios y directores de grandes industrias fueron juzgados ante el Tribunal Militar Nacional de Estados Unidos, porque apoyaron al régimen nacionalsocialista y el trabajo esclavo de civiles y prisioneros de guerra en Alemania y en los países ocupados.

Stephan Lindner, "Das Urteil im I.G.-Farben-Prozess" en NMT: Die Nürnberger Militärtribunale zwischen Geschichte, Gerechtigkeit und Rechtschöpfung, eds. Kim Christian Priemel y Alexa Stiller (Hamburgo: Hamburger Edition, 2013), 432. 
Las bases legales para estos juicios fueron la Ordenanza núm. 7 del Gobierno Militar y la Ley núm. 10 del Consejo de Control Aliado del 20 de diciembre de 1945. Los delitos estaban descritos en el artículo 2 de dicha ley. Los delitos fueron: delitos contra la paz, crímenes de guerra, crímenes de lesa humanidad y pertenencia a una organización criminal.

\subsection{El caso Flick}

El juicio contra el gran industrial Friedrich Flick y otros tuvo lugar entre el 19 de abril y el 22 de diciembre de 1947 ante el Tribunal IV Militar Nacional estadounidense en Núremberg. Los acusados fueron Friedrich Flick, Otto Steinbrinck, Bernhard Weiss, Odilo Burkart, Konrad Kaletsch y Hermann Terberger. ${ }^{5}$ Friedrich Flick era el fundador y director del consorcio Flick; Otto Steinbrinck fue el asistente de Flick; Bernhard Weiss, Odilo Burkart y Konrad Kaletsch fueron los asistentes de Flick en la oficina de Berlín, y Hermann Terberger era miembro de la junta directiva de Eisenwerkgesellschaft Maximilianshütte A. G., una importante empresa del consorcio en Baviera. ${ }^{6}$

Flick era una de las principales sociedades en Alemania, la cual poseía numerosas empresas y empleaba a miles de personas para la extracción de carbón y hierro, y la fabricación de acero, maquinaria y otros productos que utilizaban acero como materia prima. ${ }^{7}$ Fue una de las mayores productoras de armas del régimen nazi, entre ellas, granadas, piezas de artillería, tanques, bombas, aviones, piezas de submarinos, combustible y vagones. ${ }^{8}$

La acusación constó de cinco cargos: i) crímenes de guerra y crímenes de lesa humanidad, por la participación en el programa de trabajo esclavo del Tercer Reich y el uso de prisioneros de guerra en la producción de armas; ii) crímenes de guerra y crímenes de lesa humanidad por expropiación de bienes públicos y privados en territorios ocupados; iii) delitos de lesa humanidad por el saqueo de propiedades industriales mediante presiones económicas antisemitas; iv) pertenencia al círculo Keppler o amigos de Himmler y financiación de los escuadrones de protección

5 Johannes Bähr et al., Der Flick-Konzern im Dritten Reich (Múnich: Oldenbourg Wissenschaftsverlag, 2008), 622.

$6 \quad$ US Military Tribunal Nuremberg, Judgment of 22 December 1947, Flick Trial.

US Military Tribunal Nuremberg, Judgment of 22 December 1947.

8 Klaus Drobisch, "Fall 5: Der Prozess gegen Industrielle (gegen Friedrich Flick und andere)" en Der Nationalsozialismus vor Gericht: die alliierten Prozesse gegen Kriegsverbrecher und Soldaten, (1943-1952), ed. Gerd Rolf Überschar (Fráncfort del Meno: Fischer Taschenbuch Verlag, 1999), 123. 
del Partido Nacionalsocialista con conocimiento de sus actividades delictivas, y v) pertenencia a las SS, la cual era una organización criminal. ${ }^{9}$

No todos los cargos cobijaban a todos los acusados. Los implicados fueron procesados por su participación en los delitos y su posición en el consorcio Flick. Respecto a cada cargo, con excepción del quinto, se concluyó que sus acciones y conductas fueron ilegales, intencionales, con conocimiento y constituyeron violaciones de varias leyes, en particular del artículo 2 de la Ley del Consejo de Control núm. 10. ${ }^{10}$

\subsubsection{Cargo 1: participación en el programa de trabajo esclavo del Tercer Reich} y utilización de prisioneros de guerra en la producción de armas

Durante la guerra, el Tercer Reich inició un sistema de trabajo esclavo. La descripción de este programa fue tratada en su totalidad por el Tribunal Militar Internacional en el juicio de Núremberg contra las principales figuras del régimen nazi.

El esquema incluyó trabajadores civiles extranjeros, prisioneros de guerra y reclusos en campos de concentración, que fueron empleados en las fábricas del consorcio Flick trabajadores extranjeros y prisioneros de guerra. En algunas de ellas, los prisioneros de guerra desempeñaban trabajos directamente relacionados con las operaciones de guerra.

En 1944, Flick tenía más de 120000 empleados, 60000 prisioneros de guerra y trabajadores civiles extranjeros (alrededor del $40 \%$ de ellos provenientes de la Unión Soviética), casi 1000 internos de campos de concentración y al menos 80 condenados por la justicia alemana. ${ }^{11}$

El programa de trabajo esclavo tuvo su origen en los círculos de poder del Estado y fue un plan de gobierno. Los acusados no participaron en su creación ni su lanzamiento. ${ }^{12}$

Las prácticas crueles que caracterizaron el programa de trabajo esclavo en muchos lugares no prevalecieron en las fábricas e instalaciones bajo el control de los acusados. Los casos individuales de malos tratos revelados durante el juicio no fueron el

\footnotetext{
US Military Tribunal Nuremberg, Judgment of 22 December 1947.

US Military Tribunal Nuremberg, Judgment of 22 December 1947.

Drobisch, "Fall 5", 123.

12 US Military Tribunal Nuremberg, Judgment of 22 December 1947.
} 
resultado de una política de gestión del consorcio, pues estaba en contradicción directa con ella. ${ }^{13}$ Además, los acusados no tenían control o supervisión real sobre los campos de trabajo asociados a sus fábricas, ya que, entre los deberes de los demandados, como miembros de los consejos de administración de varias empresas del grupo Flick, estaba su presencia en las oficinas, en Berlín. ${ }^{14}$

Las pruebas relativas a los acusados Otto Steinbrinck, Odilo Burkart, Konrad Kaletsch y Hermann Terberger demostraron que existía un "peligro claro y presente". El Tribunal manifestó que esos cuatro acusados actuaron en un estado de necesidad: "Cuando se demuestre que el hecho imputado fue realizado para evitar un mal grave e irreparable; que no había otras rutas de escape adecuadas; y que los medios no fueran desproporcionados al mal". ${ }^{15}$

El Tribunal consideró que el régimen nazi era un reino de terror, en el cual vivían los acusados. La policía secreta estaba siempre presente y vigilaba a los alemanes en todo momento. ${ }^{16}$ Por este motivo, se reconoció el estado de necesidad de Otto Steinbrinck, Odilo Burkart, Konrad Kaletsch y Hermann Terberger, pero Friedrich Flick y Bernhard Weiss no fueron cobijados por esta justificación, porque actuaron activamente para utilizar el trabajo esclavo en su beneficio.

Bernhard Weiss, con el conocimiento y la aprobación de Friedrich Flick, estableció el aumento de la cuota de producción de vagones de mercancías, que eran equipos militares bajo la Convención de La Haya. Bernhard Weiss también ayudó a conseguir un gran número de prisioneros de guerra rusos para la fabricación de tales dispositivos. ${ }^{17}$ El esfuerzo de guerra requirió que todos los involucrados usaran todas las instalaciones para explotar completamente la producción de guerra. Este aumento no se produjo por iniciativa de los círculos gubernamentales, sino de la dirección del consorcio Flick, por lo que, para el Tribunal, ambos acusados no actuaron por coacción o miedo. ${ }^{18}$

Friedrich Flick y Bernhard Weiss fueron declarados culpables por el primer cargo, y Otto Steinbrinck, Odilo Burkart, Konrad Kaletsch y Hermann Terberger fueron absueltos.

\footnotetext{
US Military Tribunal Nuremberg, Judgment of 22 December 1947

US Military Tribunal Nuremberg, Judgment of 22 December 1947.

US Military Tribunal Nuremberg, Judgment of 22 December 1947.

US Military Tribunal Nuremberg, Judgment of 22 December 1947.

US Military Tribunal Nuremberg, Judgment of 22 December 1947.

US Military Tribunal Nuremberg, Judgment of 22 December 1947.
} 


\subsubsection{Cargo 2: expropiación de propiedad pública y privada en territorios ocupados}

Friedrich Flick y sus asistentes Bernhard Weiss, Odilo Burkart y Konrad Kaletsch explotaron las fundiciones de hierro mediante el Grupo Flick en Lorena (Francia) durante la guerra. Friedrich Flick estaba interesado en expandir su emporio y adquirir acerías adicionales. Con la ocupación alemana de Lorena, Flick vio las oportunidades que ofrecía la invasión e intentó agregar las fundiciones de hierro de esa región a su organización. Estas acciones constituyeron una violación de las Convenciones de La Haya que establecen que la propiedad privada debe ser respetada. ${ }^{19}$

Bernhard Weiss, Odilo Burkart y Konrad Kaletsch desempeñaron un papel menor en los planes de Flick. Ellos eran sus empleados y no tenían participación accionaria en la empresa. Le proporcionaron información y consejos, pero las decisiones eran suyas. No se comprometieron con algún plan de "saqueo sistemático" con Flick ni con funcionarios estatales, por lo que no se encontró falta en su conducta por la que debieran ser castigados.

El Tribunal determinó que Friedrich Flick era el único responsable del segundo cargo. Otto Steinbrinck, Bernhard Weiss, Odilo Burkart y Konrad Kaletsch fueron absueltos.

\subsubsection{Cargo 3: saqueo de propiedad mediante presión económica antisemita}

Flick utilizó su cercanía al régimen para seguir adelante con la expansión de su organización. Como socio cercano, aunque no necesariamente convencido, de líderes del partido como Göring y Himmler, Flick pudo beneficiarse del bum armamentista nazi como ningún otro empresario. Su actitud oportunista y su falta de escrúpulos se revelaron con claridad durante el curso de la guerra. Las adquisiciones de las empresas judías Hochofenwerk Lübeck y Petschek se consideraron ejemplos de "arianización" industrial. ${ }^{20}$

US Military Tribunal Nuremberg, Judgment of 22 December 1947.

20 Axel Drecoll, "Der Auftakt der Industriellen-Prozesse: Der Fall 5 gegen die Manager des Flick-Konzerns" en NMT: Die Nürnberger Militärtribunale zwischen Geschichte, Gerechtigkeit und Rechtschöpfung, eds. Kim Christian Priemel y Alexa Stiller (Hamburgo: Hamburger Edition, 2013), 378. 
Los acusados participaron en la persecución de los judíos por parte de los nazis de alguna manera e incluso aprovecharon el "programa de arianización" al buscar y utilizar la presión económica del Estado para adueñarse de las propiedades industriales de los judíos.

El Tribunal no consideró que una persona cometiera un crimen de lesa humanidad al aprovechar las presiones antisemitas para obtener la compra o la gestión de propiedades de judíos. Las conductas descritas en la sección "Crímenes de lesa humanidad" de la Ley núm. 10 son atentados contra las personas y no contra las propiedades. Las vulneraciones de derechos de propiedad industrial, por más censurables que sean, no son crímenes de ese tipo. ${ }^{21}$ Por esta razón, los imputados no fueron condenados por el tercer cargo, el cual fue desestimado.

\subsubsection{Cargo 4: pertenencia al círculo de amigos de Himmler}

El Tribunal determinó que las SS correspondían a una organización delictiva que se había utilizado con fines criminales, la cual era dirigida por Himmler. Las SS persiguieron y exterminaron judíos, cometieron brutalidades, asesinaron e impusieron el trabajo esclavo en los campos de concentración. ${ }^{22}$

Friedrich Flick y Otto Steinbrinck pertenecieron al círculo de amigos de Himmler y donaron dinero a las SS. ${ }^{23}$ Aproximadamente cuarenta personas estaban en este círculo, incluidos banqueros, industriales, funcionarios del Gobierno y miembros de las SS. Al menos la mitad de ellos contribuyó con donación de fondos. Hubo seis donaciones de 100000 marcos alemanes y la suma anual total superó el millón, de los cuales Friedrich Flick y Otto Steinbrinck aportaron 100 000.24

Friedrich Flick y Otto Steinbrinck entregaron el dinero a la organización criminal de Himmler con pleno conocimiento de las actividades criminales de las SS. ${ }^{25} \mathrm{El}$ Tribunal consideró que no importaba si este dinero era gastado en salarios o en gas letal. ${ }^{26}$ Las donaciones comenzaron mucho antes de la guerra, en un momento en el que las actividades de las SS habían comenzado, aunque no eran muy conocidas. La misma cantidad se recaudó anualmente hasta 1944 y con este dinero se mantuvo

\footnotetext{
US Military Tribunal Nuremberg, Judgment of 22 December 1947.

US Military Tribunal Nuremberg, Judgment of 22 December 1947.

US Military Tribunal Nuremberg, Judgment of 22 December 1947.

US Military Tribunal Nuremberg, Judgment of 22 December 1947.

US Military Tribunal Nuremberg, Judgment of 22 December 1947.

US Military Tribunal Nuremberg, Judgment of 22 December 1947.
} 
la asociación de Himmler. ${ }^{27}$ Debido a esto, el Tribunal condenó a Friedrich Flick y Otto Steinbrinck por el cargo 4.

\subsubsection{Cargo 5: membresía en organizaciones criminales}

Otto Steinbrinck fue un oficial naval en la Primera Guerra Mundial y por esta razón fue miembro de las SS. Durante su membresía solo tuvo dos tareas de protocolo. ${ }^{28}$ No tenía deberes ni pago y solo hacía conexiones ocasionales con los líderes de las SS. Estas actividades no lo vincularon al programa criminal de las SS; sin embargo, el Tribunal lo condenó por este cargo, al ceder voluntariamente su buena reputación a una organización cuya reputación era mala.

El Tribunal condenó a Friedrich Flick a siete años de prisión; a Otto Steinbrinck, a cinco años y a Bernhard Weiss, a dos años y medio de prisión. Odilo Burkart, Konrad Kaletsch y Hermann Terberger fueron absueltos por el tribunal.

\subsection{El caso I. G. Farben}

El juicio contra Carl Krauch y otros tuvo lugar a partir del 14 de agosto de 1947 hasta el 30 de julio de 1948 ante el VI Tribunal Militar Nacional estadounidense en Núremberg. Los acusados eran veinticuatro altos ejecutivos de I. G. Farbenindustrie AG, entre ellos, el presidente del Consejo de Supervisión, los diecinueve miembros del Consejo de Administración y cuatro directores. Carl Krauch, químico y presidente del Consejo de Supervisión, fue el principal acusado en este proceso. ${ }^{29}$

I. G. Farben se fundó en 1925 cuando, Badische Anilin und Sodafabrik en Ludwigshafen cambió su nombre a Farben y se fusionó con cinco grandes empresas químicas alemanas. En 1916 formaron un consejo para ejercer control conjunto sobre la producción, el mercado, la investigación y para aumentar sus beneficios. ${ }^{30}$

En 1926, la empresa tenía 93742 empleados y una facturación anual de 1209 millones de marcos alemanes. En 1942 empleaba a 187700 personas y facturaba

\footnotetext{
US Military Tribunal Nuremberg, Judgment of 22 December 1947.

US Military Tribunal Nuremberg, Judgment of 22 December 1947.

Lindner, "Das Urteil", 405.

US Military Tribunal Nuremberg, Judgment of 30 July 1948, I.G. Farben Trial
} 
2904 millones. En el apogeo de sus actividades, las ventas anuales superaron los 3000 mil millones. ${ }^{31}$

Farben poseía o tenía participaciones en 400 empresas alemanas y alrededor de 500 de otros países. También controlaba cerca de 40000 patentes. La acusación la describió como un "Estado sin Estado". ${ }^{32}$ Los logros de I. G. Farben en la investigación química y en el uso práctico de sus descubrimientos fueron sobresalientes. Tres ganadores del Premio Nobel eran científicos de este consorcio. ${ }^{33}$ I. G. Farben producía nitrógeno, combustibles sintéticos, lubricantes, carbón, tintes, metales ligeros, productos químicos, productos farmacéuticos, fibras sintéticas, celulosa, celofán y materiales fotográficos. ${ }^{34}$

La empresa y el régimen se unieron como un matrimonio por conveniencia: debido a la competencia internacional, I. G. Farben necesitó el apoyo del Reich y los lucrativos contratos estatales y, para llevar a cabo la guerra, Hitler requería combustible, caucho y explosivos. ${ }^{35} \mathrm{El}$ consorcio se benefició de la autosuficiencia, del armamento, de la agresión, de la explotación y de las políticas de exterminio del Reich. ${ }^{36}$

Durante la Segunda Guerra Mundial, I. G. Farben saqueó y se apropió de bienes extranjeros en Alemania y los territorios ocupados, suministró pesticida Zyklon B a los campos de concentración y exterminio, se benefició de experimentos humanos en campos de concentración y empleó a numerosos trabajadores forzados al final de la guerra; por ejemplo, el campo de concentración de Monowitz (Auschwitz III) fue construido alrededor de la fábrica de Buna, propiedad de I. G. Farben, para tener trabajadores disponibles para el trabajo industrial. ${ }^{37}$

La acusación constaba de cinco cargos: i) delitos contra la paz mediante la planificación, preparación, iniciación y realización de guerras de agresión e invasiones a otros países; ii) crímenes de guerra y de lesa humanidad al participar en el saqueo de bienes públicos y privados en países y territorios que quedaron bajo la ocupación

\footnotetext{
US Military Tribunal Nuremberg, Judgment of 30 July 1948.

US Military Tribunal Nuremberg, Judgment of 30 July 1948.

US Military Tribunal Nuremberg, Judgment of 30 July 1948.

US Military Tribunal Nuremberg, Judgment of 30 July 1948.

35 Florian Jessberger, "Die IG Farben vor Gerichtt: Von den Ursprüngen eines Wirtschaftsvölkerstrafrechts", Juristenzeitung, núm. 19 (2009): 924.

36 Jessberger, "Die IG Farben vor Gericht", 925.

37 Jessberger, "Die IG Farben vor Gericht", 925.
} 
militar de Alemania; iii) crímenes de guerra y de lesa humanidad por la participación en la esclavitud y el trabajo forzado de la población civil en países y zonas ocupadas o controladas por Alemania, así como la participación en la esclavización de prisioneros en campos de concentración en Alemania y el uso de prisioneros de guerra en operaciones militares y trabajo ilegal. Además, maltrato, terror, tortura y asesinato de personas esclavizadas; iv) pertenencia a una organización criminal, y v) participación en una conspiración para cometer delitos contra la paz. ${ }^{38}$

1.2.1. Cargos 1 y 5: delitos contra la paz por planificar, preparar, iniciar y llevar a cabo guerras de agresión e invasiones a otros países, y participación en una conspiración para cometer crímenes contra la paz

Los cargos 1 y 5 se basaron en los mismos hechos y contienen la misma evidencia. Por lo tanto, se estudiaron conjuntamente. El Tribunal abordó con gran cautela la decisión de condenar a un acusado por los cargos de participación en un plan conjunto o conspiración, o de planear y llevar a cabo una guerra de agresión. Desde este punto de vista, la evidencia tanto del conocimiento como de la participación fue contundente. ${ }^{39}$

No estaba claro cuándo Hitler concibió su plan de agresión o con quién lo discutió. En una reunión secreta, el 15 de noviembre de 1937, hizo una revelación final; sin embargo, ninguno de los acusados asistió a estas reuniones. ${ }^{40}$

La evidencia demostró que los acusados no participaron en la planificación de la guerra de agresión. Los planes fueron hechos dentro de un círculo celosamente vigilado: las reuniones fueron secretas, la información intercambiada fue confidencial y los acusados estaban por fuera de este círculo, por lo que no tuvieron oportunidad de participar en la planificación, la preparación o el inicio de la guerra. ${ }^{41}$

El Tribunal concluyó que no eran culpables ${ }^{42}$ y los absolvió por estos cargos.

\footnotetext{
US Military Tribunal Nuremberg, Judgment of 30 July 1948.

US Military Tribunal Nuremberg, Judgment of 30 July 1948.

US Military Tribunal Nuremberg, Judgment of 30 July 1948.

US Military Tribunal Nuremberg, Judgment of 30 July 1948.

US Military Tribunal Nuremberg, Judgment of 30 July 1948.
} 


\subsubsection{Cargo 2: crimenes de guerra y de lesa humanidad por participación en el} saqueo de propiedad pública y privada

Entre el 12 de marzo de 1938 y el 8 de mayo de 1945, los representantes de I. G. Farben participaron en el saqueo de propiedad pública y privada en Polonia, Francia, Alsacia-Lorena, Noruega y Rusia. ${ }^{43}$ El consorcio recurrió al saqueo sistemático de propiedad extranjera al hacerse con el control de determinadas empresas, aunque en muchas ocasiones se mantuvo la apariencia de que se estaba pagando por la propiedad confiscada. ${ }^{44}$ En la mayoría de los casos fue una iniciativa de la sociedad alemana. ${ }^{45}$

Los acusados actuaron contra el artículo 7, párrafo 1, literal b de la Ley del Consejo núm. 10 y los artículos 46, 47, 53 y 55 de la Convención de La Haya de 1907. El Tribunal declaró que la propiedad pública y privada debía mantenerse intacta durante la ocupación militar.

Ernst Bürgin, Paul Häfliger, Max Ilgner, Friedrich Jähne, Hans Kugler, Fritz ter Meer, Heinrich Oster, Hermann Schmitz y Georg von Schnitzler participaron en la acción ilegal. Conocieron y aprobaron la expropiación de fábricas en Polonia, Francia, Alsacia-Lorena y Noruega y, como resultado, estos acusados fueron declarados culpables y los demás fueron puestos en libertad.

\subsubsection{Cargo 3: crímenes de guerra y de lesa humanidad por participación en la esclavitud y el trabajo forzado de personas}

I. G. Farben participó en el programa de trabajo esclavo del Tercer Reich, suministró gas venenoso para exterminar a los reclusos de los campos de concentración, entregó drogas para experimentos médicos criminales en individuos esclavizados y participó en prácticas inhumanas en su planta industrial en Auschwitz. ${ }^{46}$

Este programa utilizó una enorme reserva de trabajo esclavo. Muchas de estas personas fueron utilizadas en actividades relacionadas con operaciones militares

US Military Tribunal Nuremberg, Judgment of 30 July 1948.

Jessberger, "Die IG Farben vor Gericht", 927.

4 US Military Tribunal Nuremberg, Judgment of 30 July 1948.

46 US Military Tribunal Nuremberg, Judgment of 30 July 1948 
contra su propio país, en violación directa del derecho internacional, y en actividades industriales y agrícolas. ${ }^{47}$

El Tribunal declaró que Hermann Röchling, Otto Heinrich von Gemmingen y Rodenhauser no actuaron en un estado de emergencia; por el contrario, explotaron el trabajo esclavo de jóvenes rusos, franceses, belgas y holandeses ${ }^{48} \mathrm{y}$, en consecuencia, fueron declarados culpables por este cargo.

El campo de concentración de Auschwitz fue financiado y era propiedad de I. G. Farben. Ambrós, Buetefisch y Dürrfeld fueron los responsables de la construcción en Auschwitz. ${ }^{49}$ Allí se introdujo el trabajo forzado de polacos, prisioneros de guerra y reclusos. El uso de prisioneros de guerra en condiciones inhumanas constituyó una violación de la Convención de Ginebra y un crimen de guerra. El uso de trabajadores de campos de concentración y trabajadores forzados extranjeros en Auschwitz fue un crimen de lesa humanidad y un crimen de guerra. ${ }^{50}$

Los miembros de I. G. Farben fueron responsables de tales crímenes, ya que el empleo de trabajadores de campos de concentración en Auschwitz fue una iniciativa suya. Además, los acusados también fueron conscientes de los abusos y los tratos inhumanos hacia los internos. ${ }^{51}$

Por otro lado, Degesch, una filial de I. G. Farben, suministró grandes cantidades del pesticida Zyclon B a las SS, que se utilizó en el exterminio masivo de reclusos en los campos de concentración. ${ }^{52}$ El Tribunal concluyó que los acusados conocían estos hechos, pero no sabían del uso delictivo de esta sustancia. ${ }^{53}$

Los reclusos sanos fueron infectados deliberadamente con tifus en los campos de concentración en contra de su voluntad para que I. G. Farben probara sus medicamentos y muchos de ellos murieron a causa de la enfermedad. ${ }^{54}$ El Tribunal declaró a los acusados inocentes, porque no tenían conocimiento de los experimentos

\footnotetext{
47 US Military Tribunal Nuremberg, Judgment of 30 July 1948.

48 US Military Tribunal Nuremberg, Judgment of 30 July 1948.

49 US Military Tribunal Nuremberg, Judgment of 30 July 1948.

50 US Military Tribunal Nuremberg, Judgment of 30 July 1948.

51 US Military Tribunal Nuremberg, Judgment of 30 July 1948.

52 US Military Tribunal Nuremberg, Judgment of 30 July 1948.

3 US Military Tribunal Nuremberg, Judgment of 30 July 1948.

54 US Military Tribunal Nuremberg, Judgment of 30 July 1948.
} 
ilegales. ${ }^{55}$ Carl Krauch, Otto Ambros, Heinrich Bütefisch, Walter Dürrfeld y Fritz ter Meer fueron declarados culpables; los demás fueron puestos en libertad.

\subsubsection{Cargo 4: pertenencia a una organización criminal}

Schneider, Büterfisch y Von der Heyde se convirtieron en miembros del escuadrón de protección del Partido Nacionalsocialista de los Trabajadores Alemanes (SS) ${ }^{56}$ después del 1 de septiembre de 1939. El Tribunal declaró que esta organización era criminal.

Schneider, Büterfisch y Von der Heyde eran miembros de las SS, pero no había pruebas de que tuvieran conocimiento de las actividades delictivas de la organización y por eso fueron absueltos por este cargo.

El Tribunal condenó a Carl Krauch a seis años de prisión; a Otto Ambros, a ocho años; a Walter Dürrfeld, a ocho años; a Fritz ter Meer, a siete años; a Georg von Schnitzler a siete años; a Heinrich Bütefisch, a seis años; a Hermann Schmitz, a cuatro años; a Max Ilgner, a tres años; a Heinrich Oster, a dos años; a Ernst Bürgin, a dos años; a Paul Häfliger, a dos años; a Friedrich Jähne, a un año y medio, y a Hans Kugler, a un año y medio. Los otros acusados fueron absueltos.

\subsection{El caso Krupp}

El juicio contra el industrial Alfried Krupp von Bohlen y Halbach y otros tuvo lugar entre el 8 de diciembre de 1947 y el 31 de julio de 1948 ante el Tribunal III Militar Nacional estadounidense en Núremberg.

Los acusados fueron Alfried Krupp von Bohlen y Halbach, Karl Eberhardt, Eduard Houdremont, Max Ihn, Friedrich Janssen, Heinrich Korschan, Hans Kupke, Heinrich Lehmann, Ewald Löser, Erich Müller y Karl Pfirsch. Krupp fue acusado por los actos de su padre, Gustav, puesto que sufría de demencia avanzada y no podía ser juzgado. ${ }^{57}$

US Military Tribunal Nuremberg, Judgment of 30 July 1948.

US Military Tribunal Nuremberg, Judgment of 30 July 1948.

Kim Christian Priemel, "Der Sonderweg vor Gerichtt. Angewandte Geschichte im Nürnberger Krupp-Prozess", Historische Zeitschrift 294, núm. 2 (2012): 391, DOI: https://doi.org/10.1524/hzhz.2012.0015 (acceso mayo 8, 2021). 
El consorcio Krupp fue fundado en 1812 y era propietario o controlaba minas y fábricas de acero y armamento, como la de acero fundido Grusonwerk A. G. y el astillero Germania. ${ }^{58}$ El grupo fue uno de los mayores fabricantes de armas del régimen nazi, ${ }^{59}$ así como placas de blindaje, cañones, cascos de tanques, torretas blindadas, granadas, barcos y submarinos. ${ }^{60}$

La acusación constó de cuatro cargos: i) planificación, preparación, iniciación y ejecución de una guerra de agresión; ii) saqueo; iii) delitos contra prisioneros de guerra y trabajo forzado, y iv) conspiración para cometer delitos contra la paz. ${ }^{61}$

Los imputados fueron absueltos por los cargos 1 y 4 , pues el tribunal concluyó que la evidencia no mostraba más allá de toda duda que los acusados fueran culpables de estos delitos. ${ }^{62}$

\subsubsection{Cargo 2: saqueo}

Krupp saqueó fábricas en Francia y Países Bajos, en gran parte, de la manera más obvia y directa, al retirar máquinas y materiales que envió a Alemania. Además, en otras formas recurrió a cambios en la propiedad de la empresa y transferencia contractual de derechos de propiedad. ${ }^{63}$

Estos actos violaron las leyes y costumbres de la guerra, los tratados y las convenciones internacionales, incluidos los artículos 46 a 56 de la Convención de La Haya de 1907 y el artículo 2 de la Ley núm. 10 del Consejo de Control. ${ }^{64}$

La adquisición de inmuebles, máquinas y materiales en los países ocupados fue una iniciativa de esa firma. ${ }^{65}$ Un testigo declaró que, el 18 de mayo de 1940, Alfried Krupp y otros tres industriales se habían reunido en una mesa para revisar un mapa y escuchar una transmisión por radio de noticias de guerra alemanas. Al final del reporte, los cuatro hombres señalaron con el dedo ciertos lugares del

\footnotetext{
58 US Military Tribunal Nuremberg, Judgment of 31 July 1948, Krupp Trial.

59 Priemel, "Der Sonderweg vor Gerichtt", 395.

60 US Military Tribunal Nuremberg, Judgment of 31 July 1948.

61 US Military Tribunal Nuremberg, Judgment of 31 July 1948.

62 US Military Tribunal Nuremberg, Judgment of 31 July 1948.

63 US Military Tribunal Nuremberg, Judgment of 31 July 1948.

64 US Military Tribunal Nuremberg, Judgment of 31 July 1948.

65 US Military Tribunal Nuremberg, Judgment of 31 July 1948.
} 
mapa que indicaban pueblos y fábricas. Uno dijo: "Este es tuyo, este es tuyo [...] tiene dos fábricas". ${ }^{66}$

El Tribunal concluyó que Krupp, Loeser, Houdremont, Müller, Janssen y Eberhardt eran culpables del cargo 2 y los demás fueron puestos en libertad.

\subsubsection{Cargo 3: delitos contra prisioneros de guerra y trabajo esclavo}

Krupp participó en el programa de trabajo esclavo del régimen nazi. Toda la empresa, que constaba de alrededor de 81 plantas separadas en el área metropolitana de Alemania, empleó a un total de 69898 trabajadores civiles extranjeros y 4978 prisioneros de campos de concentración entre 1940 y 1945. La gran mayoría de ellos fue llevada a la fuerza a Alemania y detenida bajo coacción durante todo su período de servicio, al igual que 23076 prisioneros de guerra. ${ }^{67}$

La empresa empleó a prisioneros de guerra de Francia, Bélgica, Holanda, Polonia, Yugoslavia, Rusia e Italia en la producción de armas contra sus propios países. ${ }^{68}$ Las condiciones en los campos de concentración de Krupp eran terribles. Los prisioneros estaban hambrientos, eran maltratados, no tenían una vivienda adecuada ni instalaciones médicas reales y trabajaban demasiado tiempo. Este acto constituyó una violación del derecho internacional.

El estado de emergencia no fue aceptado como defensa de los acusados, pues el Tribunal determinó que el uso de mano de obra en campos de concentración fue voluntario y no una imposición del régimen sobre el grupo Krupp. Los miembros del consorcio conocieron los vejámenes cometidos en los campos de concentración, de los cuales se beneficiaron. ${ }^{69}$

Krupp, Loeser, Houdremont, Mueller, Janssen, Ihn, Eberhardt, Korschan, Von Buelow, Lehmann y Kupke fueron declarados culpables, mientras Pfirsch fue absuelto por este cargo. Por último, el Tribunal condenó a Alfried Krupp von Bohlen y Halbach, Friedrich von Bülow y Erich Müller a doce años de prisión; a Eduard Houdremont y Friedrich Janssen, a diez años; a Karl Eberhardt y Max Ihn, a nueve años; a Ewald Löser, a siete años; a Heinrich Korschan y Heinrich 
Lehmann, a seis años y a Hans Kupke, a dos años, diez meses y diecinueve días. Karl Pfirsch fue absuelto.

\section{Criterios para evaluar si los empresarios fueron responsables por la comisión de crímenes de guerra y de lesa humanidad}

El Tribunal Militar estadounidense definió varios criterios en los juicios posteriores de Núremberg contra los industriales para juzgar si ellos participaron en la comisión de crímenes de guerra y de lesa humanidad.

Los elementos de decisión establecidos en las sentencias Flick, I. G. Farben y Krupp son los siguientes:

1. Las sanciones penales del derecho internacional se aplican a los particulares, no solo a los funcionarios estatales.

2. La explotación económica en los territorios ocupados está limitada por los convenios de La Haya y Ginebra.

3. No basta con que el empresario sea miembro de la junta directiva de la compañía; es necesario que el acusado participara en un delito determinado o aprobara su comisión.

4. Ciertos comportamientos impiden la aplicación de la defensa por estado de emergencia.

5. Se requiere una acción activa o un conocimiento concreto de la conspiración para iniciar una guerra de agresión.

\subsection{Las sanciones penales del derecho internacional se aplican a los particulares, no solo a los funcionarios}

El Tribunal juzgó a funcionarios estatales; sin embargo, en los casos revisados se procesó a personas que no ocupaban cargos públicos ni representaban al Estado. ${ }^{70}$

70 US Military Tribunal Nuremberg, Judgment of 22 December 1947. 
No había razón para limitar la responsabilidad de los oficiales, puesto que las acciones consideradas criminales seguían siendo criminales incluso si las cometió un particular. ${ }^{71}$

En estos casos, la mayoría de los acusados no estaba afiliada oficialmente al Gobierno nazi. Los procesados eran particulares que se desempeñaban como empresarios de la industria pesada alemana y fueron condenados conforme al derecho penal internacional. ${ }^{72}$ Por otro lado, el Tribunal también mencionó que las empresas no habían sido juzgadas como personas jurídicas. ${ }^{73}$

\subsection{La explotación económica en los territorios ocupados está limitada por los convenios de La Haya y Ginebra}

Los directores de Flick, I. G. Farben y Krupp cometieron crímenes de guerra y de lesa humanidad según el derecho de La Haya y de Ginebra, pues apoyaron el programa de trabajo esclavo del régimen nazi, utilizaron prisioneros de guerra como trabajadores en sus fábricas y saquearon industrias en los territorios ocupados.

Las empresas alemanas que utilizaron fábricas y mano de obra local en dichos territorios para apoyar la economía de guerra alemana cometieron crímenes de guerra en virtud de las Convenciones de La Haya de 1907. De acuerdo con su artículo 52, la economía en tales zonas debe mantenerse intacta, excepto cuando las autoridades de ocupación otorgan permisos cuidadosamente definidos. Sus residentes no deben ser obligados a ayudar al enemigo a librar la guerra contra su propio país. ${ }^{74}$

Asimismo, violaron el derecho de La Haya cuando adquirieron fábricas en los territorios ocupados o se apoderaron de sus máquinas para enviarlas a Alemania. Los artículos 46 y 47 de la Convención de La Haya de 1907 prohíben la confiscación de propiedad privada y el saqueo.

Por último, los empresarios también violaron la Convención de Ginebra de 1927, porque obligaron a los prisioneros de guerra a trabajar como mano de obra esclava en sus fábricas. El uso de prisioneros de guerra en los campos de concentración e

US Military Tribunal Nuremberg, Judgment of 30 July 1948.

US Military Tribunal Nuremberg, Judgment of 22 December 1947.

US Military Tribunal Nuremberg, Judgment of 30 July 1948.

74 US Military Tribunal Nuremberg, Judgment of 31 July 1948. 
instalaciones en condiciones inhumanas es una violación de los artículos 29 y 30 del Convenio de Ginebra de 1929, "Convenio III de Ginebra sobre el trato debido a los prisioneros de guerra" y el artículo 6 de los Convenios de La Haya de 1907.

\subsection{No basta con que el empresario sea miembro de la junta directiva de la compañía; es necesario que el acusado participe en un delito determinado o apruebe su comisión}

Los directores de Flick, I. G. Farben y Krupp fueron declarados culpables de cometer crímenes de guerra o contra la humanidad porque participaron, tenían conocimiento o aprobaron actividades delictivas dentro de sus empresas.

Los miembros condenados de la junta directiva de las empresas implicadas ordenaron, aprobaron, planearon o actuaron en el programa de saqueo y trabajo esclavo adelantado por el régimen nazi. También sabían de la persecución a los judíos y de las atrocidades cometidas en los campos de concentración y en las fábricas que administraban. ${ }^{75}$

Algunos miembros de la junta directiva fueron absueltos por no asistir o por desconocer la comisión de crímenes de guerra y de lesa humanidad por parte de la empresa.

\subsection{Ciertos comportamientos impiden la aplicación de la defensa por estado de emergencia}

Todos los acusados en los tres juicios dijeron que habían actuado en un estado de emergencia. El Tribunal Militar examinó a fondo este eximente de responsabilidad, basó el estado de emergencia en la ley estadounidense y descartó su aplicación en tres situaciones.

El Tribunal manifestó:

El estado de emergencia es una defensa cuando se demuestra que el hecho imputado fue realizado para evitar un mal grave e irreparable; que no existían

75 US Military Tribunal Nuremberg, Judgment of 31 July 1948. 
otras vías de escape adecuadas; y que los medios no eran desproporcionados frente al posible mal. ${ }^{76}$

Además, determinó que los acusados estaban en "peligro claro y presente" debido al reinado del terror del Reich. Ellos vivían en el régimen, el cual siempre estuvo presente con sus funcionarios y la policía secreta los monitoreaba. ${ }^{77}$

El Tribunal no aceptó que algunos procesados se defendieran con el estado de emergencia, puesto que tomaron medidas activas para aumentar la producción utilizando prisioneros de guerra o mano de obra esclava en sus fábricas, incluso más allá de lo ordenado por el Gobierno nazi ${ }^{78} \mathrm{y}$, en consecuencia, no actuaron bajo coacción de las autoridades del Reich.

En el estado de emergencia es importante que "el acto imputado se hiciera para evitar un mal grave e irreparable" y "que los medios no fueran desproporcionados al mal". No había proporcionalidad entre la posibilidad de que los industriales perdieran sus trabajos y el control de sus propiedades frente a emplear a miles de civiles deportados, prisioneros de guerra y reclusos en campos de concentración. ${ }^{79}$ Tampoco era posible que los industriales hubieran sido llevados a un campo de concentración, porque tenían influencia y amistad con los líderes nazis y sus empresas tenían un gran poder en Alemania. ${ }^{80}$

\subsection{Se requiere una acción activa o un conocimiento concreto de la conspiración para iniciar una guerra de agresión}

Para que los imputados fueran declarados culpables por un delito contra la paz por participar en la planificación, la preparación y el inicio de guerras de agresión o invasión, se debía demostrar que conocían el plan o la conspiración, su finalidad y el fin promovido.

El Tribunal concluyó que no era claro cuándo Hitler ideó su plan de agresión o con quién lo discutió por primera vez. Los empresarios no participaron en la planificación de la guerra de agresión, los planes fueron hechos por y dentro de

US Military Tribunal Nuremberg, Judgment of 22 December 1947.

US Military Tribunal Nuremberg, Judgment of 22 December 1947.

US Military Tribunal Nuremberg, Judgment of 22 December 1947.

US Military Tribunal Nuremberg, Judgment of 31 July 1948.

US Military Tribunal Nuremberg, Judgment of 31 July 1948. 
un círculo celosamente restringido y las reuniones fueron secretas. Los industriales estaban muy por debajo de los miembros de este círculo y no tuvieron oportunidad de participar en la planificación, la preparación o el inicio de la guerra. ${ }^{81}$

Los miembros de las empresas alemanas solo sabían del rearme de Alemania, pero desconocían el plan de la guerra de agresión. Debido a esto fueron absueltos por el cargo de conspiración de guerra de agresión. ${ }^{82}$

\section{Críticas a los juicios de Núremberg contra los industriales}

El juicio de los principales criminales de guerra de Núremberg y los juicios posteriores de Núremberg recibieron críticas. La defensa manifestó que estos fueron una expresión de la "justicia de los vencedores", porque los jueces y la Fiscalía eran representantes de los aliados y también afirmó que el Tribunal había violado el principio nullum crimen sine lege. ${ }^{83}$ Otras posturas se refirieron a la regulación de los justificantes como el estado de emergencia en el estatuto del Tribunal y el tratamiento de los delitos de organización. Durante el juicio, la defensa acusó a los aliados del bombardeo de áreas de ciudades alemanas por parte de unidades británicas y estadounidenses. ${ }^{84}$

Los juicios posteriores de Núremberg contra los industriales también fueron reprobados. Incluso, décadas después, los fiscales consideraron que la sentencia fue demasiado indulgente y estaban decepcionados por la cuantía de las sentencias y las absoluciones. En 1951, todos los condenados fueron indultados. ${ }^{85}$

Además, de los industriales, los banqueros trabajaron en estrecha colaboración con el régimen nazi. Aunque estaban gravemente comprometidos, no fueron juzgados. ${ }^{86}$

81 US Military Tribunal Nuremberg, Judgment of 30 July 1948.

82 US Military Tribunal Nuremberg, Judgment of 30 July 1948.

83 Academia Internacional de los Principios de Núremberg, "Von Nürnberg nach Den Haag: Lehren aus der Vergangenheit-Herausforderungen für die Zukunft", https://www.nurembergacademy.org/fileadmin/media/ pdf/Non_Nuernberg_nach_Den_Haag.pdf (acceso mayo 5, 2021).

84 Academia Internacional de los Principios de Núremberg, "Von Nürnberg nach Den Haag".

85 Lindner, "Das Urteil", 433

86 Jonathan Wiesen, "Die Verteidigung der deutschen Wirtschaft. Nürnberg, das Industriebüro und die Herausbildung des Neuen Industriellen" en NMT: Die Nürnberger Militärtribunale zwischen Geschichte, Gerechtigkeit und Rechtschöpfung, eds. Kim Christian Priemel y Alexa Stiller (Hamburgo: Hamburger Edition, 2013), 631. 
Alfried Krupp von Bohlen und Halbach fue juzgado en lugar de su padre, porque Gustav Krupp padecía de demencia. Su incapacidad para ser procesado había escapado a los equipos de acusación estadounidenses y británicos y solo se hizo pública por su abogado defensor. Este error vergonzoso obedeció a que los preparativos de la fiscalía fueron apresurados. ${ }^{87}$

En las sentencias no hay declaraciones de la Fiscalía o del Tribunal sobre la calificación precisa del rol de los imputados (como perpetradores o como partícipes). Esto fue sorprendente porque el artículo 2 de la Ley núm. 10 del Consejo de Control Aliado diferenciaba las formas de participación, al menos conceptualmente. ${ }^{88}$

\section{La importancia de los juicios de Núremberg contra los industriales en casos como el colombiano}

Los juicios contra los principales criminales nazis en Núremberg dejaron enseñanzas para el derecho penal internacional. ${ }^{89}$ Por su parte, los juicios posteriores de Núremberg contra los industriales dan luces para analizar casos del derecho penal internacional, ${ }^{90}$ en los cuales los actores económicos participaron en la comisión de crímenes de guerra y de lesa humanidad.

Los procesos contra los industriales permitieron iniciar un proceso de reconciliación con el pasado. ${ }^{91}$ Las sentencias mostraron su participación en los crímenes nazis, ${ }^{92}$ pues ellos aceptaron gradualmente las ideas de política económica del régimen por convicción, oportunismo o una mezcla de ambos. ${ }^{93}$ En determinadas circunstancias, algunos industriales se sintieron obligados a someterse a los dictados del Gobierno nacionalsocialista. ${ }^{94}$

87 Priemel, "Der Sonderweg vor Gerichtt", 438

88 Jessberger, "Die IG Farben vor Gericht", 929

89 Christoph Safferling, "Lernen von Nürnberg", Rechtsgeschichte 6, núm 14 (2009): 148, http://data.rg.mpg. de/rechtsgeschichte/rg14_148safferling.pdf (acceso mayo 8, 2021).

90 Miriam Saage-Maaß, "Geschäft ist Geschäft? Zur Haftung von Unternehmen wegen der Förderung staatlicher Menschenrechtsverletzungen", Kritische Justiz 1, núm. 43, (2010): 57, https://www.jstor.org/stable/24240471 (acceso mayo 8, 2021).

91 Wiesen, "Die Verteidigung", 632.

92 Grietje Baars, "Capitalism's Victor's Justice? en The Hidden Story of the Prosecution of Industrialists Post-WWll" en The Hidden Histories of War Crimes Trials, eds. Kevin Jon Heller and Gerry Simpson (Oxford: Oxford University Press, 2013), 165.

93 Avraham Barkai, "Die deutschen Unternehmer und die Judenpolitik im Dritten Reich", Geschichte und Gesellschaft 15, núm. 2 (1989): 227, https://aggb-katalog.de/vufind/Record/dok.45814 (acceso mayo 8, 2021).

94 Wiesen, "Die Verteidigung", 632. 
Estos juicios posteriores establecieron los requisitos para determinar la responsabilidad penal de la acción empresarial de acuerdo con el derecho penal internacional y por primera vez intentaron abarcar de manera integral la actividad empresarial con los instrumentos que este ofrecía. Gracias a estos procesos se ha desarrollado el método de estudio de la macrocriminalidad y han sido la piedra angular del derecho penal económico internacional. ${ }^{95}$

La práctica del derecho penal internacional se concentra en enjuiciar a las personas que han participado en la comisión de crímenes de derecho internacional, como funcionarios, militares o políticos; sin embargo, la responsabilidad de las empresas comerciales o sus empleados en el mejor de los casos, ha sido tratada por tribunales nacionales e internacionales, pese a que se ha indicado reiteradamente que los intereses económicos a menudo han sido un motor para la comisión de crímenes de derecho internacional. ${ }^{96}$

La Fiscalía colombiana investiga a 15291 civiles involucrados en la comisión de crímenes de lesa humanidad y de guerra durante el conflicto armado. Se trata de ciudadanos que pertenecen a corporaciones y empresas con presencia en las regiones de confrontaciones. ${ }^{97}$ Entre estos, 1253 son actores económicos, investigados por delitos relacionados con promoción, asistencia o financiamiento de grupos armados ilegales que han cometido crímenes de guerra y de lesa humanidad. ${ }^{98}$ Se conoce la investigación a exejecutivos y empleados de la empresa Chiquita Brands, que financió a grupos paramilitares. ${ }^{99}$

Desde junio de 2004, la Fiscalía de la Corte Penal Internacional (CPI) ha estado indagando la situación en Colombia como parte del examen preliminar, de conformidad con el artículo 15, párrafo 1, del Estatuto de Roma. La fiscal Fatou Bensouda ha indicado que la jurisprudencia del derecho penal internacional es relevante a la hora de investigar y enjuiciar a empresarios. ${ }^{100}$

95 Jessberger, "Die IG Farben vor Gericht", 931.

96 Jessberger, "Die IG Farben vor Gericht", 931.

97 Nelson Camilo Sánchez León y Daniel Marín López, "Corporate Accountability in Transitional Justice in Colombia" en Peace, Everyone's Business! Corporate Accountability in Transitional Justice: Lessons for Colombia, eds. Joris van de Sandt y Marianne Moor (Utrecht: PAX, 2017), 125.

98 Corte Penal Internacional, "Report on Preliminary Examination Activities 2019", https://www.icc-cpi.int/ itemsDocuments/191205-rep-otp-PE.pdf (acceso septiembre 30, 2021).

99 Corte Penal Internacional, "Report on Preliminary".

100 Corte Penal Internacional, "Escrito de Amicus Curiae de la fiscal de la Corte Penal Internacional sobre la Jurisdicción Especial para la Paz ante la Corte Constitucional de la República de Colombia", http:// 
Asimismo, la Jurisdicción Especial para la Paz (JEP) debe juzgar a los civiles que voluntariamente se sometan a ella, conforme con el artículo 63 de la Ley estatutaria de la administración de justicia en la JEP. Muchos de ellos son actores económicos que participaron en la comisión de crímenes internacionales, para lo cual será necesario utilizar la jurisprudencia internacional como elemento interpretativo, con el fin de analizar su responsabilidad personal.

Es común que los actores económicos manifiesten haber participado en la comisión de crímenes graves por coacción. ${ }^{101}$ Por tanto, sería fundamental seguir los criterios fijados en los juicios de Núremberg contra los industriales y determinar si los empresarios actuaron cobijados por la eximente de responsabilidad del estado de necesidad, el cual se encuentra descrito en el artículo 32 del Código Penal colombiano.

En este contexto, los juicios contra industriales en Núremberg serían guías para el análisis del caso colombiano, en particular respecto a la imputación de responsabilidad penal a empresarios y su participación en la comisión de crímenes internacionales durante el conflicto armado.

\section{Conclusiones}

Los juicios posteriores de Núremberg fueron personales, en contra de criminales nazis. Estos juicios tuvieron lugar después del final de la Segunda Guerra Mundial en las zonas ocupadas por Francia, Gran Bretaña, Estados Unidos y la Unión Soviética en Alemania; sin embargo, los doce juicios posteriores de Núremberg adelantados en la zona ocupada estadounidense son los más conocidos.

Estos últimos tuvieron la particularidad de haber sido divididos en profesiones y gremios dentro del Gobierno nazi. En efecto, los acusados fueron catalogados como miembros del régimen nazi, la Administración, el Ejército, el Poder Judicial, la industria y las SS. Su fundamento jurídico fue la Ordenanza núm. 7 del Gobierno Militar y la Ley núm. 10 del Consejo de Control Aliado del 20 de diciembre de 1945.

Los juicios de Núremberg contra los industriales (Flick, I. G. Farben y Krupp) son la piedra angular del derecho penal económico internacional, toda vez que fueron

cr00.epimg.net/descargables/2017/10/21/17135b6061c7a5066ea86fe7e37ce26a.pdf?int=masinfo (acceso septiembre 30, 2021).

101 Alejandro Jiménez Ospina, Hobeth Martínez Carrillo y Daniel Marín López, Los terceros complejos: la competencia de la Jurisdicción Especial para la Paz (Bogotá: Dejusticia, 2019), 68. 
los primeros grandes procesos adelantados en contra de empresarios y gerentes de la industria pesada alemana y fueron condenados según el derecho penal internacional.

La relación entre la industria alemana y el régimen nazi quedó expuesta en estos juicios. Las sentencias mostraron la participación de la gran industria alemana en los crímenes nazis, pues se comprobó que los acusados, por medio de sus empresas, apoyaron al régimen nazi, financiaron las SS y participaron en la comisión de crímenes internacionales.

Las industrias de Flick, I. G. Farben y Krupp produjeron armamento durante la Segunda Guerra Mundial, tuvieron una estrecha cooperación con el líder de las SS, Heinrich Himmler, y otros prominentes nacionalsocialistas, saquearon propiedades en los territorios ocupados y recurrieron al uso forzoso de civiles, prisioneros de guerra y reclusos en campos de concentración e industrias en toda Europa. Debido a esto fueron juzgados y condenados por crímenes de guerra y de lesa humanidad.

Los juicios posteriores de Núremberg tuvieron dos méritos: establecieron los requisitos para determinar la responsabilidad del actuar criminal de industriales, de conformidad con el derecho penal internacional, y develaron los patrones de macrocriminalidad de la acción empresarial con los instrumentos de las normas del derecho penal internacional.

Los requisitos para determinar la responsabilidad del actuar criminal de los industriales, de conformidad con el derecho penal internacional, que fueron definidos por el Tribunal Militar Nacional estadounidense en los juicios posteriores de Núremberg adelantados contra los industriales alemanes fueron los siguientes:

En primer lugar, las sanciones penales del derecho internacional incluyen a los particulares, no solo a los funcionarios. En efecto, en los casos Flick, I. G. Farben y Krupp se procesaron a personas que no ocupaban cargos públicos ni representaban al Estado; eran particulares que se desempeñaban como empresarios de la industria pesada alemana y fueron juzgados y condenados conforme al derecho penal internacional.

En segundo lugar, los convenios de La Haya y Ginebra sirvieron de base para condenar a los industriales por la explotación económica en los territorios ocupados. Los directores de Flick, I. G. Farben y Krupp cometieron crímenes de guerra y de lesa humanidad según el derecho de La Haya y de Ginebra, pues apoyaron el 
programa de trabajo esclavo del régimen nazi, utilizaron prisioneros de guerra como trabajadores en sus fábricas y saquearon industrias en los territorios ocupados.

En tercer lugar, no basta con que el empresario sea miembro de la junta directiva de la compañía, sino que es necesario que participe en un delito determinado o apruebe su comisión. Así, los directores de Flick, I. G. Farben y Krupp fueron declarados culpables de cometer crímenes de guerra o contra la humanidad, porque participaron, tenían conocimiento o aprobaron actividades delictivas dentro de sus empresas. Por un lado, los miembros condenados de la junta directiva de las empresas implicadas ordenaron, aprobaron, planearon o actuaron en el programa de saqueo y trabajo esclavo adelantado por el régimen nazi. Además, sabían de la persecución a los judíos y conocieron las atrocidades cometidas en los campos de concentración y en las fábricas que administraban. Por otro lado, se demostró que los miembros absueltos no aprobaron o desconocieron la comisión de crímenes de guerra y de lesa humanidad por parte de sus empresas.

En cuarto lugar, ciertos comportamientos impiden recurrir la defensa por estado de emergencia. El Tribunal Militar examinó a fondo este eximente de responsabilidad y descartó su aplicación en tres situaciones: i) cuando los acusados tomaron medidas activas para aumentar la producción utilizando prisioneros de guerra o mano de obra esclava en sus fábricas, incluso más allá de lo ordenado por el Gobierno nazi; ii) no existiera proporcionalidad entre la amenaza del régimen contra los empresarios y los graves crímenes que ellos cometieron en los territorios ocupados y en campos de concentración, y iii) las consecuencias por la no colaboración de los procesados con el Tercer Reich no eran tan graves, puesto que los acusados tenían influencia y amistad con los líderes nazis y sus empresas tenían un gran poder en Alemania.

Por último, para declarar la responsabilidad por un delito de conspiración para iniciar una guerra de agresión es requerida una acción o un conocimiento concreto de la conspiración; por tal razón, los acusados fueron absueltos por este delito, por cuanto ellos no conocieron el plan de agresión ideado por la cúpula nazi.

Los juicios posteriores de Núremberg contra los empresarios son relevantes para determinar la responsabilidad penal de los empresarios en otros casos, con base en los criterios señalados en el presente documento y para ilustrar la relación entre los intereses económicos y la comisión de crímenes de derecho internacional, por ejemplo, el de Colombia, en donde se investigan 1253 actores económicos 
por delitos relacionados con promoción, asistencia o financiamiento de grupos armados ilegales que han cometido crímenes de guerra y de lesa humanidad.

\section{Referencias}

Academia Internacional de los Principios de Núremberg. "Von Nürnberg nach Den Haag: Lehren aus der Vergangenheit-Herausforderungen für die Zukunft". https://www. nurembergacademy.org/fileadmin/media/pdf/Non_Nuernberg_nach_Den_Haag.pdf (acceso mayo 5, 2021).

Andoor, George. "Das Nürnberger Tribunal vor 70 Jahren-Teil 2. Faires Verfahren anhand der Grundsätze eines neuen Völkerstrafrechts". Zeitschrift für das Juristische Studium 5, núm. 8 (2015): 473-484, http://www.zjs-online.com/dat/artikel/2015_5_939.pdf (acceso mayo 8, 2021).

Baars, Grietje. "Capitalism's Victor's Justice? The Hidden Story of the Prosecution of Industrialists Post-WWll" en The Hidden Histories of War Crimes Trials, editado por Kevin Jon Heller and Gerry Simpson, 163-192. Oxford: Oxford University Press, 2013.

Bähr Johannes, Axel Drecoll, Bernhard Gotto, Kim Christian Priemel y Harald Wixforth. Der Flick-Konzern im Dritten Reich. Múnich: Oldenbourg Wissenschaftsverlag, 2008.

Barkai, Avraham. "Die deutschen Unternehmer und die Judenpolitik im Dritten Reich". Geschichte und Gesellschaft 15, núm. 2 (1989): 227-247, https://aggb-katalog.de/vufind/ Record/dok.45814 (acceso mayo 8, 2021).

Corte Penal Internacional. "Escrito de Amicus Curiae de la fiscal de la Corte Penal Internacional sobre la Jurisdicción Especial para la Paz ante la Corte Constitucional de la República de Colombia". http://cr00.epimg.net/descargables/2017/10/21/171 35b6061c7a5066ea86fe7e37ce26a.pdf?int=masinfo (acceso septiembre 30, 2021).

Corte Penal Internacional. "Report on Preliminary Examination Activities 2019". https:// www.icc-cpi.int/itemsDocuments/191205-rep-otp-PE.pdf (acceso septiembre 30, 2021).

Drecoll, Axel. "Der Auftakt der Industriellen-Prozesse: Der Fall 5 gegen die Manager des Flick-Konzerns" en NMT: Die Nürnberger Militärtribunale zwischen Geschichte, Gerechtigkeit und Rechtschöpfung, editado por Kim Christian Priemel y Alexa Stiller, 376-404. Hamburgo: Hamburger Edition, 2013.

Drobisch, Klaus. "Fall 5: Der Prozess gegen Industrielle (gegen Friedrich Flick und andere)" en Der Nationalsozialismus vor Gericht: die alliierten Prozesse gegen Kriegsverbrecher und Soldaten, (1943-1952), editado por Gerd Rolf Überschar, 121-132. Fráncfort del Meno: Fischer Taschenbuch Verlag, 1999.

Jessberger, Florian. "Die IG Farben vor Gericht: Von den Ursprüngen eines Wirtschaftsvölkerstrafrechts". Juristenzeitung, núm. 19 (2009): 924-932. 
Jiménez Ospina, Alejandro, Hobeth Martínez Carrillo y Daniel Marín López. Los terceros complejos: la competencia de la Jurisdicción Especial para la Paz. Bogotá: Dejusticia, 2019. Lindner, Stephan. "Das Urteil im I.G.-Farben-Prozess" en NMT: Die Nürnberger Militärtribunale zwischen Geschichte, Gerechtigkeit und Rechtschöpfung, editado por Kim Christian Priemel y Alexa Stiller, 405-433. Hamburgo: Hamburger Edition, 2013.

Priemel, Kim Christian. "Der Sonderweg vor Gericht. Angewandte Geschichte im Nürnberger Krupp-Prozess", Historische Zeitschrift 294, núm. 2 (2012): 391-426, DOI: https:// doi.org/10.1524/hzhz.2012.0015 (acceso mayo 8, 2021).

Saage-Maaß, Miriam. "Geschäft ist Geschäft? Zur Haftung von Unternehmen wegen der Förderung staatlicher Menschenrechtsverletzungen" Kritische Justiz 1, núm. 43 (2010): 54-61, https://www.jstor.org/stable/24240471 (acceso mayo 8, 2021).

Safferling, Christoph. "Der Nürnberger Prozess" en Krieg und Recht. Die Ausdifferenzierung des Rechts von der ersten Haager Friedenskonferenz bis heute, editado por Martin Löhnig, Mareike Preisner y Thomas Schlemmer, 87-98. Regenstauf: Rechtskultur, 2014.

Safferling, Christoph. "Lernen von Nürnberg", Rechtsgeschichte 6, núm 14 (2009): 148-161, http://data.rg.mpg.de/rechtsgeschichte/rg14_148safferling.pdf (acceso mayo 8, 2021).

Safferling, Christoph. Internationales Strafrecht: Strafanwendungsrecht-VölkerstrafrechtEuropäisches Strafrecht. Heidelberg: Springer, 2011.

Sánchez León, Nelson Camilo y Daniel Marín López. "Corporate Accountability in Transitional Justice in Colombia" en Peace, Everyone's Business! Corporate Accountability in Transitional Justice: Lessons for Colombia, editado por Joris van de Sandt y Marianne Moor, 120-140. Utrecht: PAX, 2017.

US Military Tribunal Nuremberg. Judgment of 22 December 1947. Flick Trial.

US Military Tribunal Nuremberg. Judgment of 30 July 1948. I.G. Farben Trial.

US Military Tribunal Nuremberg. Judgment of 31 July 1948. Krupp Trial.

Wiesen, Jonathan. "Die Verteidigung der deutschen Wirtschaft. Nürnberg, das Industriebüro und die Herausbildung des Neuen Industriellen" en NMT: Die Nürnberger Militärtribunale zwischen Geschichte, Gerechtigkeit und Rechtschöpfung, editores Kim Christian Priemel y Alexa Stiller, 630-652. Hamburgo: Hamburger Edition, 2013. 